\title{
Automatic summarization of medical interviews
}

\author{
Jipeng Qiang* \\ Department of computer science, Yangzhou University, Yangzhou, Jiangsu, China
}

\begin{abstract}
The genomic-based targeted therapy (Crizotinib) has been emerged as an alternative option for the treatment of patients with locally advanced or metastatic non-small cell lung cancer, comprising the $85 \backslash \%$ of lung cancer. However, Crizotinib is not listed in VA drug formulary and is not available for VA oncologists to treat lung cancer currently. Therefore, for understanding physicians' views on using genomic services, semistructured interviews were collected. In this paper, we will present an innovative method to extract summarization from medical interviews automatically. Different from keyword-based method, automatic summarization can help to understand the intention of physicians. Compared with the existing summarization methods, our work is based on latent Dirichlet allocation and recent results in word embeddings that learn semantically meaningful representations for words from local cooccurrences in sentences. Experiments on medical interviews demonstrate that the proposed algorithm achieves good results compared with a gold standard file using manual extraction technique.
\end{abstract}

\section{Introduction}

Lung cancer is the second most common cancer in the United States, with an estimated 221,130 new cases in 2011 [1]. Due no cost-effective screening for lung cancer in practice, lung cancer is difficult to detect at an early stage [1]. Physicians play a key role for patients in terms of access to genomic-based targeted treatment. Older physicians were likely to order more tests, while younger physicians were likely to adhere to the recommended practice guidelines $[2,3]$. Therefore, for understanding physicians' views on using genomic services, semi-structured interviews were conducted with a large of physicians. Knowledge gained from the interviews will provide valuable information for VA policy decisionmakers to understand the importance of providing genotyping-guided care to veterans with advanced lung cancer.

Womack et al. in their study of extracting fracture events from VA radiology reports in the Veterans Aging Cohort Study using the NegEx algorithm [4]. The NegEx is conceptbased, not keyword-based, indexing for information extraction. A major limitation of keyword-based data extraction is that it is associated with limited utility (low sensitivity and PPV) of extracting information from clinical narratives. The key word search can only determine whether the term or terms exist or not, and its performance completely depends on whether queries and documents happen to choose the exact same terms to express the

\footnotetext{
* Corresponding author: jpqiang@yzu.edu.cn
} 
same concepts. On the other hand, even if text fragments containing search terms are retrieved, it does not mean that these texts refer to the concepts requested by users due to polysemy and ambiguity of natural languages. In summary, the terms extracted by key word-based indexing cannot provide sufficient and reliable information for critical decision-making in genetic testing. The NegEx algorithm is designed to solve the problem associated with keyword information extraction by linking EGFR mutation with negative or no through a natural language processing approach. In this study, we will present automatic analysis and summarization of spoken medical dialogue. Different from Keyword extraction, automatic summarization deals with whole sentences instead of words and phrases that can help to understand the intention of physicians.

Existing summarization methods mainly fall into two categories: extractive summarization and abstractive summarization [5]. The former directly extracts the most informative document components (e.g. sentence), and abstractive summarization involves reformulation of contents. Extractive summarization is a simple but robust method without the requirement for advanced post-processing steps, such as centroid-based methods, graphbased methods, pattern-based methods, and topic-based methods. In this paper, we only focus on extractive summarization. Centroid-based methods group document sentences into homogeneous clusters, and then select the representative sentences through computing the similarity values between sentences and the centroids of the clusters [6]. Graph-based methods [7] construct a graph, where the nodes correspond to document sentences and edges correspond to relation (usually some kind of similarity or distance), and then propagate sentence information on the graph according to some criteria. Topic-based methods [8-9] select the representation sentences using the latent topics in the documents discovered by topic modelings. Considering the problems of polysemy and synonymy, some ontology-based methods [10] have also been used to generate summaries using lexical semantics. But they are restricted in some specific application domains where ontologies are available.

To overcome these inherent weaknesses and keep the advantages of both term-based and ontology-based methods, we propose a novel method, Embedding-based summarization (EBS), to extract summarization from spoken medical dialogue. Our method leverages recent results by word embeddings that obtain vector representations for words [11]. EBS has the following three steps. EBS firstly builds distributed word embeddings from a large corpus. Then EBS clusters all sentences using latent Dirichlet allocation algorithm [12-14]. Finally, EBS chooses the most representative sentences from each clusters by incorporating the semantic knowledge from word embeddings. Experiments on medical demonstrate that the proposed algorithm achieves good results compared with a gold standard file using manual extraction technique.

\section{Method}

In this section, we discuss our method, Embedding-based Summarization(EBS), for extracting a summary underlying a collection of dialogues. First, we cluster all sentences using latent Dirichlet allocation algorithm. Third, we select the most representative sentences from each clusters by incorporating the semantic knowledge from word embeddings which utilizes a new metric, Word Mover's Distance (WMD) [15], to compute the distance between two short text.

\subsection{Sentence Clustering}

Before the information extraction step, we will conduct a pre-text processing on Medical Interviews. The pre-text process includes a series of steps: (1) Convert letters into 
lowercase; (2) Remove non-latin characters and stop words; (3) Remove words whose length are smaller than 3 or larger than 20; (4) Remove words with frequency less than 3. The existing NLP tools [16] is used to split semi-structured interviews into sentences. After pre-processing, suppose a sentence collection $\mathrm{S}=\left\{\mathrm{s}_{1}, \mathrm{~s}_{2}, \ldots, s_{l}, s_{N}\right\}$, where $s_{l}$ represents the $l$ th sentence of $S, N$ is the number of sentences in $S$. Each sentence $s l \in \mathrm{S}$ consists of a set of words, where $s_{l i}$ represents the $i$ th word of sentence $s_{l}$. Latent Dirichlet Allocation (LDA) is used to estimate hidden variables $z_{l i}$, where $\mathbf{Z}_{l i}$ denotes the topic identities assigned to the $i$ th word $s_{l i}$. LDA uses collapsed Gibbs Sampling to estimate parameters under Dirichlet priors $\alpha$ and $\beta$, and the conditional distribution is given by,

$$
\mathrm{p}\left(z_{l i}=k \mid S^{-l i}, \alpha, \beta\right) \propto \frac{\left(n_{l}^{k,-l i}+\alpha\right)\left(n_{k}^{w_{i l},-l i}+\beta\right)}{n_{k}^{-l i}+V \beta}
$$

where $z_{l i}$ refers to topic $k$ of the $i$ th word $w_{l i}$ in the $l$ th sentence of $S, \mathrm{p}\left(z_{l i}=k \mid S^{-l i}, \alpha, \beta\right)$ is the probability of word $\underline{w}_{l i}$ belonging to topic $k$ conditioned on the whole set $S$ after removing the current word, $n^{k}$ is the number of occurrences of topic $k$ in sentence $l, n_{k}^{w_{l i}}$ is the number of occurrences of word $\underline{w}$ li belonging to topic $k$ in $S, n_{k}$ is the number of occurrences of all words $W$ belonging to topic $k$ in $S$, and $V$ is the size of $W$. Moreover, the superscript.$^{-l i}$ means topic $k$ of word $w_{l i}$ in sentence $l$ is removed from $z_{l}$. Here, $K$ is the number of topics in $S$, and $k=\{1,2, \ldots, K\}$. For instance, $n_{k}^{-l i}$ is obtained through removing the (topic, word) combination at the ith word of the lth sentence.

Finally, using the counts of topic assignments of sentences, we can estimate the sentence-topic distribution $\theta$ as follows,

$$
\phi_{k}^{\mathrm{s}_{l}}=\frac{n_{k}^{s_{l}}+\alpha}{n_{s_{l}}+K \alpha}
$$

where $n_{s_{l}}$ is the number of words in text $s_{l}$, and $n_{k}^{s_{l}}$ is the number of words belonging to topic $k$ in text $s l$.

After obtaining $N \times K$ matrix $\theta$, we use matrix $\theta$ to determine the cluster label of each sentence. More precisely, we need to examine each row $l$ of matrix $\theta$, and assign sentence $s l$ to cluster $k$ if $k=\arg \max _{k=1}^{K} \theta_{l k}$.

\subsection{Sentence Selection}

To select the most pertinent and meaningful sentences into the summary, we select one sentence from each clustering that owns the highest similar to its clustering and less similar to other clusterings.

Let $\left\{C_{1}, C_{2}, \ldots, C_{k}, \ldots, C_{K}\right\}$ represent the sentences of the $K$ clustering, respectively, where $\left|C_{k}\right|$ represent the number of sentences in clustering $C_{k}$. Let $\mathrm{s}_{k}^{i}$ be the $i$ th sentence in clustering $C_{k}$. Hence, the score of the sentence $\mathrm{s}_{k}^{i}$ is defined as follows:

$$
\operatorname{score}\left(s_{k}^{i}\right)=\lambda \frac{\sum_{j \in C_{k,-i}} \operatorname{Sim}\left(s_{k}^{i}, s_{k}^{j}\right)}{\left|C_{k}\right|}-(1-\lambda) \frac{\sum_{j \in C_{k,-i}} \operatorname{Sim}\left(s_{k}^{i}, s_{j}\right)}{N-\left|C_{k}\right|}
$$

where $\operatorname{Sim}\left(s_{i}, s_{j}\right)$ is the similarity between sentence $s_{i}$ and $s_{j}, \lambda$ is a user-specified parameter that controls the trade-off between inner-cluster coverage and outer-clustering redundancy, $" j \in C_{k},-i \$ "$ means all sentences in clustering $C_{k}$ except sentence $\mathrm{s}_{k}^{i}$, and $" \mathrm{j} \in \mathrm{S},-C_{k}$ " means all sentences in $S$ except all sentences in $C_{k}$. Below, we will explain how to compute the similarity between sentences by incorporating words embeddings. 
After obtaining word embeddings of each word, we use the typical cosine distance measure for the distance between words. We assume each sentence is represented as normalized bag-of-words (nBOW) vector, $\mathbf{r}_{\mathrm{i}} \in \mathrm{R}^{\mathrm{V}}$, a V-dimension vector, $r_{i, w}=\frac{c_{i, w}}{\sum_{j=1}^{V} c_{i, j}}$ where $c_{i, w}$ denotes the occurrence times of word $w$ in text $s_{i}$. We can see that an BOW vector is very sparse as only a few words appear in each text. Therefore, we introduce a new metric, called the Word Mover's Distance\} (WMD), to compute the distance between texts. WMD computes the minimum cumulative cost that words from one sentence need to travel to match exactly the words of the other sentence as the distance of sentences, in which the distance between words is computed by word embeddings.

\section{Experiments}

\subsection{Datasets Description}

Semi-structured interviews were conducted with 20 providers working in different units at the Health Care System. The contents of interviews were tape-recorded, transcribed and content analyzed using qualitative methods. The interviews can be used to assess patients behavioral, normative, and control beliefs about the delivery of genomic medicine.

Table 1. A gold standard file using manual extraction technique on interviews.

\begin{tabular}{|c|c|c|}
\hline \multirow{4}{*}{ Testing } & 1 & Oncologists test for mutation at varying rates: $20-95 \%$ \\
\hline & 2 & Mutations are very rare \\
\hline & 3 & Variable understanding of testing guidelines \\
\hline & 4 & System-level barriers to testing \\
\hline \multirow{7}{*}{ Treatment } & 5 & All have used TT, most experience with erlotinib \\
\hline & 6 & Most describe guideline-concordant care \\
\hline & 7 & Treatment goals: palliation of symptoms and extending life \\
\hline & 8 & Variable beliefs about efficacy of targeted therapy \\
\hline & 9 & Perceived advantages: less toxicity, convenience, efficacy \\
\hline & 10 & $\begin{array}{l}\text { Perceived disadvantages: cost, toxicity, adherence, interactions, limited } \\
\text { efficacy }\end{array}$ \\
\hline & 11 & Patients attitudes toward targeted therapy \\
\hline $\begin{array}{c}\text { Communicat } \\
\text { ion }\end{array}$ & 12 & Providers take different approaches to educating patients about TT \\
\hline Expectations & 13 & Providers expect more targeted agents to emerge in the future \\
\hline \multirow{3}{*}{$\begin{array}{l}\text { Palliative } \\
\text { Care }\end{array}$} & 14 & Referral decisions vary \\
\hline & 15 & Patients do not always react well to palliative care referrals \\
\hline & 16 & $\begin{array}{l}\text { More likely to refer patients with little or no social support, those with } \\
\text { emotional difficulty dealing with diagnosis }\end{array}$ \\
\hline
\end{tabular}

The analysis from the semi-structured interviews will include identification of broad categories related to: (1) knowledge, (2) attitudes, (3) intent to use genomic-based targeted therapy, and (4) facilitators and barriers to using genomic-based targeted therapy. To ensure the accuracy of the automated data summarization from medical interview, oncologist generated a gold standard file using manual extraction technique that is shown in Table 1. We compare our model EBS with the exist method SumBasic [17], a summarization system 
that exploits frequency exclusively to create summaries. The user-specified parameter $\lambda$ is set to 0.3 . For each automatic summarization, we only choose 10 sentences, and then match the results with the gold summarization. If the sentence of the summarization can match any sentence of the gold summarization, we will give the index, else is labeled "None".

Table 2 and Table 3 show results for EBS and Sumbasic, respectively. We can see our model EBS can identify more useful sentences compared with the gold standard file. In conclusion, our model produces better results compared to the baselines, which demonstrate the effectiveness of our model in exploring word correlation knowledge from words embeddings to improve the quality of topic modeling.

Table 2. The results using our method EBS

\begin{tabular}{|c|c|}
\hline Label & Summarization \\
\hline 5 & Crizotinib for ALK um positivity and um erlotinib or gefitinib for EGFR um. \\
\hline 6 & When we called palliative care, they were known as death doctors \\
\hline 4 & We do not test squamous, and we do not test any other mixed test status. \\
\hline None & There are different perceptions or misperceptions, which we discuss and try to remove. \\
\hline None & $\begin{array}{l}\text { Anything from a piece of tissue from lung cancer or suspected lung cancer is submitted } \\
\text { to pathology. }\end{array}$ \\
\hline 6 & $\begin{array}{l}\text { At quarterly conferences, we had to teach primary care providers, nurses, } \\
\text { chaplains, physicians. }\end{array}$ \\
\hline None & Surgeon, radiation therapist, 2 or 3 medical oncologists, and pathologists are there. \\
\hline 12 & $\begin{array}{l}\text { Then we tell them, and I mean some of them dont come to us and some do come for } \\
\text { the reading, that it is curable or not curable. }\end{array}$ \\
\hline None & $\begin{array}{l}\text { There attending, but they are not so full-time, so I think I reflect most } \\
\text { of the approaches or strategy, yeah. }\end{array}$ \\
\hline 9 & They've shown improvement, and I think they've shown more in maintenance therapy \\
\hline
\end{tabular}

Table 3. The results using Sumbasic.

\begin{tabular}{|c|c|}
\hline Label & Summarization \\
\hline 7 & Every time I talk to a patient with stage IV lung cancer,I start with palliative care. \\
\hline None & Not really you know I mean these drugs work. \\
\hline None & Targeted therapies and recently immunotherapy is the major thing. \\
\hline None & Well like I said I mean those people who are metastatic we- we test. \\
\hline 5 & We started on erlotinib for EGFR mutation positive. \\
\hline 7 & Palliate symptoms related to disease or treatments. \\
\hline None & Improving quality of life; prolonging survival \\
\hline None & It kind of makes them fulfill that patient role. \\
\hline None & When we talk about chemo, we tell them the side effects. \\
\hline None & Um nothing has changed in small cell lung cancer. \\
\hline
\end{tabular}

\section{Conclusion}

Medical interviews on physicians for lung cancer treatment is a valuable source of information for understanding physicians' views on using genomic services. This work presents a first step towards automatic analysis and summarization of spoken interviews. In this paper, we propose a novel method for automatic summarization based on latent Dirichlet allocation and words Embedding, namely Embedding-based summarization (EBS). EBS can capture more contextual semantic information than individual terms by 
incorporating the high quality of the word2vec embedding. Experimental results have shown that EBS outperforms one existing method on medical interviews datasets. In the future, we plan to train word2 $\mathrm{vec}$ on existing medical corpus.

\section{Acknowledgments}

This research is partially supported by the the National Natural Science Foundation of China under grant 61703362, Natural Science Foundation of Jiangsu Province of China under grant BK20170513, and the Natural Science Foundation of the Higher Education Institutions of Jiangsu Province of China under grant 17KJB52004.

\section{References}

1. A. C. Society, Cancer Facts \& Figures 2009, American Cancer Society, (2010)

2. R. S. Stafford, B. Misra, Variation in routine electrocardiogram use in academic primary care practice, Archives of internal medicine 161,19:2351-2355 (2001)

3. K. C. Stange, T. Fedirko, S. J. Zyzanski, C. R. Jaen, How do family physicians prioritize delivery of multiple preventive services?, Journal of Family Practice 38, 3: 231-238 (1994)

4. C. Gibert, M. Yin, A. C. Justice, M. Cynthia Brandt MD, A comparison of two approaches to text processing: facilitating chart reviews of radiology reports in electronic medical records, Perspectives in health information management, 1 (2010)

5. J. Qiang, P. Chen, W. Ding, F. Xie, X. Wu, Multi-document summarization using closed patterns, Knowledge-Based Systems, 99: 28-38 (2016)

6. D. R. Radev, H. Jing, M. Sty's, D. Tam, Centroid-based summarization of multiple documents, Information Processing \& Management 40, 6:919-938 (2004)

7. G. Erkan, D. R. Radev, Lexrank: Graph-based lexical centrality as salience in text summarization, Journal of Artificial Intelligence Research, 22, 457-479 (2004)

8. R. Arora, B. Ravindran, Latent dirichlet allocation based multi-document summarization, in: Proceedings of the second workshop on Analytics for noisy unstructured text data, ACM, 91-97 (2008)

9. J. Qiang, P. Chen, T. Wang, X. Wu. Topic Modeling over Short Texts by Incorporating Word Embeddings. PAKDD (2017)

10. E. Baralis, L. Cagliero, S. Jabeen, A. Fiori, S. Shah, Multi-document summarization based on the yago ontology, Expert Systems with Applications, 40,17, (2013)

11. T. Mikolov, K. Chen, G. Corrado, J. Dean, Efficient estimation of word representations in vector space, arXiv preprint arXiv:1301.3781 (2013)

12. D. M. Blei, A. Y. Ng, M. I. Jordan, Latent dirichlet allocation, the Journal of machine Learning research 3, 993-1022 (2003)

13. J. Qiang, P. Chen, W. Ding, T. Wang, F. Xie, X. Wu. Topic Discovery from Heterogeneous Texts. ICTAI (2016)

14. J. Qiang, Y. Li, Y. Yuan, X. Wu. Short text clustering based on Pitman-Yor process mixture model. Applied Intelligence (2017)

15. M. J. Kusner, Y. Sun, N. I. Kolkin, K. Q. Weinberger, From word embeddings to document distances, ICML (2015)

16. C. D. Manning, M. Surdeanu, J. Bauer, J. R. Finkel, S. Bethard, D. McClosky, The stanford corenlp natural language processing toolkit., in: ACL (2014)

17. A. Nenkova, L. Vanderwende, The impact of frequency on summarization, Microsoft Research, Redmond, Washington, Tech. Rep. MSR-TR-2005-101 (2005) 